\title{
Adding Analog-like Sensation to Drawing Software by Drawing Sounds
}

\author{
Yulana Watanabe *, Takayuki Fujimoto *
}

\begin{abstract}
Today, with the spared of the Internet, most of the everyday-life components are being digitized. Drawing software is an example of such developments. There are many types of software products, from drawing/painting software that can be easily used by children, to those with specific features that creators and professionals can use for delicate works. However, there are few packages of software that can give the feeling of drawing. It is assumed that the "real feel", which is easily obtained when people draw in reality leads to the improvement of work efficiency. There are many factors that enable people to obtain this feeling. In this study, we specifically focus on one of them, "Sound". It is conceivable that the generated sound varies depending on the combination of the drawing tool and the surface material, and also on the characteristics such as the writing pressure and writing speed of each user. As the first part of the series of the study, we will recognize how the sounds are differed and verify that people can obtain "sensation of drawing" by sounds
\end{abstract}

Keywords: Analog, Digital, Drawing, Feeling.

\section{Background}

\subsection{Introduction}

Today, any kinds of devices and environments are digitized. The purpose of digitizing devices and environments is to improve work efficiency and diversify expressions. Being supported by computers, people can work more efficiently than by conventional analog equipment. In addition, various new expressions, which were once considered difficult to realize in analog, have become easier. For example, "Drawing Software" classified as design software/art software is one of the digital tools to provide such support. Essentially, its function is enabling user to draw pictures with computers. Since it is implemented as digital software, it can also provide the creative operations and expressions that were difficult in analog.

\footnotetext{
* Graduate School of Information Sciences and Arts, Toyo University, Tokyo, Japan
} 


\subsection{Digitization of Drawing}

In recent years, the evolution and diversification of drawing software is remarkable. Since 1975 when the world's first paint software, "BitRectEditor" was launched, it developed rapidly. Thereafter, from Adobe, "Illustrator" in 1987, and "Photoshop" in 1989 were released, and now digital paintings are spreading more than analog paintings. Many types have been released, from software packages that children can pick up casually for scribbling, to those that is used by professionals and functions as work items. Although there are many packages of drawing software for various purposes, each of them mainly focuses on the appearance of the works and the detailed features, there are almost no software products that can give users the sensation of drawing that is usually obtained when drawing in reality. The presence or absence of "real feel" has a great influence on our work steps. It can change the work efficiency and relevantly cause the users' attachment feeling to the tools that affects the quality of the works.

\subsection{Analog-like Experience with Sounds}

There are many factors to cause the feeling of drawing, and one of the components that have a great weight is "sound." For example, when drawing in art lessons or classes, we can hear the friction sound made by a pencil with a sharpened tip and paper in a quiet space. Regarding today's drawing software products, many of them are particular about reproducibility of appearance of the analog works and subdivision of functions, and there have been few approaches to the environment and tools taking the actual work operations of drawing into consideration. In this study, we focus on the strong sensation users have when working in analog, and we assume that incorporating the analog-like reality into digital leads to the improved work efficiency and quality of work. As a means for that, we focus on the sounds generated by drawing operations.

\section{Purpose}

Today, the pen tools included in the drawing software products have been devised in various ways to enhance expressiveness. However, even if they have visual expressiveness and changes due to operations, it is difficult to give the "sensation of drawing" to the users. On the other hand, when using paper and drawing tools in analog, the "real feel" of writing can be obtained firmly from the sound, texture, and pressure. In particular, it is well known that subtle changes in the generated sound due to the combination of drawing tool and surface material in analog drawing, have a significant effect on the rhythm and pace of the creative production. As is obvious in the sound of markers, which some people hate to hear, each sound is so distinctive. The sound also changes depending on the surface material. For example, when plain paper and drawing paper are compared, drawing paper makes users feel its roughness and experience a real feeling of drawing more easily. Because of this quality, drawing paper is more commonly used for children's drawings. In this study, we first focus on the sound factor in the drawing software and conduct the following two experiments in order to enhance user's realistic experience and examine the possibility of improving user motivation and work efficiency. First, we will define the differences in the "working sounds" generated by the combination of various drawing tools and surface materials. Secondly, we will clarify whether the working sound generated by the combination of the drawing tool and the surface material makes a difference in the user experience. 


\section{Experiment 1: Changes in the Sound by Combination of Draw- ing Tool and Surface Material}

\subsection{Outline of Experiment 1}

In this study, the sound heard during the work changes depending on the drawing tool being used, and it is thought that the sound delivers a realistic experience to users. This chapter clarifies by experiments how the sound actually changes by the combination of drawing tool and surface material. There are various types of "real feeling" users get from drawing sounds generated by the combination of art materials. Therefore, in this study, three most common types of drawing tools, brush, marker and pencil are used. Then, we prepare two types of pencils: sharpened pencils with pointed tips and slightly-used ones with rounded tips. As surface material, two types of paper, plain paper and drawing paper, are used. Combinations of three types of drawing tools and two types of surface materials are recorded to compare their differences, using waveforms. For recording, the same space and desk were used so that no environmental conditions would cause any errors. In addition, the experiments were conducted with the utmost effort to use average handwriting pressure and speed to avoid any sound errors caused by differences in them.

\subsection{Changes in Sounds Caused by Combinations}

During the experiment, six letters " $\mathrm{ABC}$ abc" were written in this order and the writing sound was recorded. By visualizing the recorded sound source in waveforms, the differences will be clearly shown.

\subsubsection{Brush and high-quality plain paper, brush and drawing paper}

Figure1 show waveforms of the recorded sound generated by combining high-quality paper / drawing paper and brush as drawing tool.

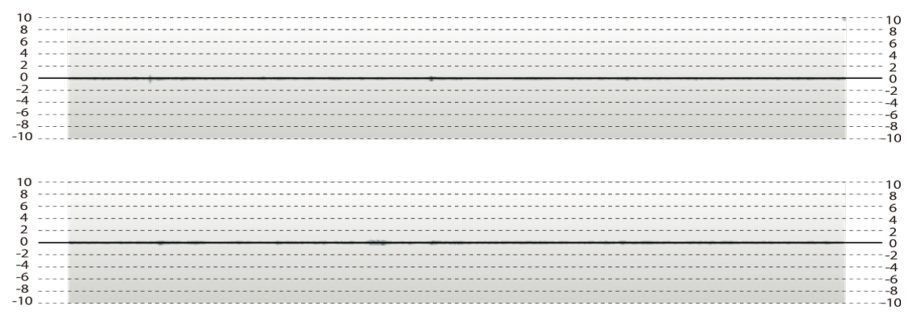

Figure1: Brush and high-quality plain paper (upper) and brush and drawing paper (lower)

There were almost no changes in the drawing sound by surface material when brush was used. In either case, the sound is so soft that it is almost inaudible unless you have a quiet environment. The sound looks generally louder when drawn on drawing paper than on high quality plain paper. At the same time, with drawing paper, ink decreases quickly and a rough sound of brush rubbing against the paper is heard.

\subsubsection{Sharpened pencil and high-quality plain paper, sharpened pencil and drawing pa- per}

Figure2 show waveforms of the recorded sound generated by combining high-quality paper / drawing paper and sharpened pencil as drawing tool. 


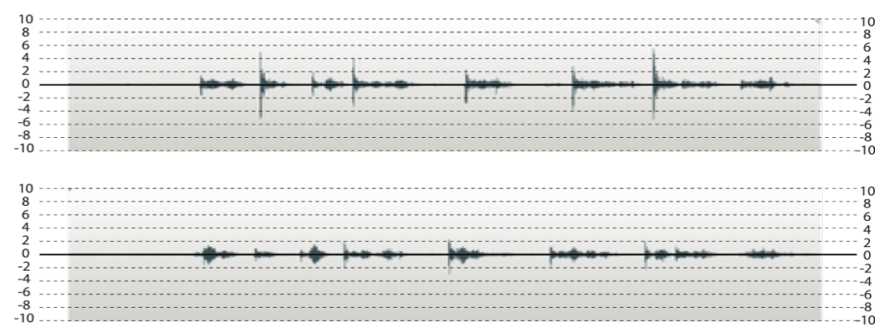

Figure2: Sharpened pencil and high-quality plain paper (upper) and sharpened pencil and drawing paper (lower)

The volume of the sound generated by drawing paper is generally more constant. No significant changes in the volume can be observed between the sound you make at the first stroke and that of the following strokes. On the other hand, when plain paper is used, the sound is relatively loud at the first stroke. However, when drawing lines that follow, the volume is not much different from that on drawing paper.

\subsubsection{Used pencil with a rounded tip and high-quality plain paper, used pencil with} a rounded tip and drawing paper

Figure3 show waveforms of recorded sound generated by combining high-quality paper / drawing paper and used pencil with a rounded tip as drawing tool. With either surface material, the first stroke tends to be loud, and the volume of subsequent lines varies. Overall, the sound volume is more constant with drawing paper. The high-quality plain paper generally produces a little louder sound, but when drawing a curvy line, the drawing paper makes a slightly louder sound.

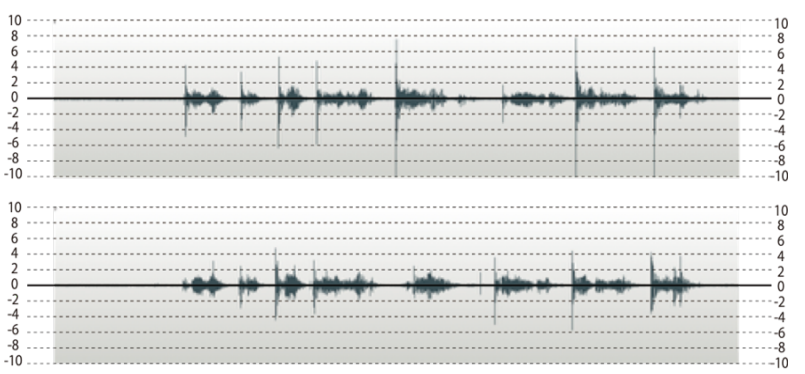

Figure3: Used pencil with a rounded tip and high-quality plain paper (upper) and used pencil with a rounded tip and drawing paper (lower)

\subsubsection{Marker and high-quality plain paper, marker and drawing paper}

Figure4 show waveforms of recorded sound generated by combining high-quality paper / drawing paper and marker as drawing tool.

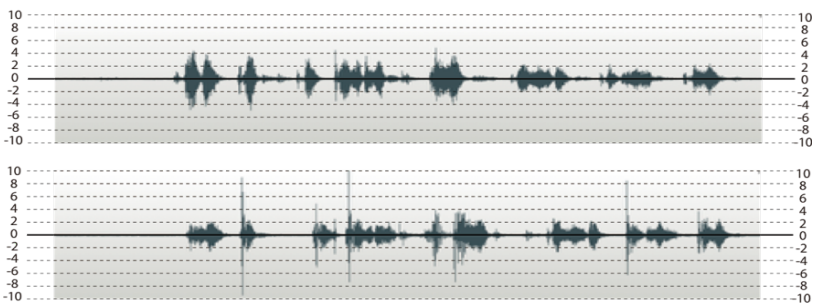

Figure4: Marker and high-quality plain paper (upper) and marker and drawing paper (lower) 
With drawing paper, variation in sound volume is smaller, but overall it is loud. With high-quality plain paper, the variation in sound volume is so large as to show a clear difference between the first stroke and the subsequent lines. The sound of the first stroke is louder when the marker is paired with high quality plain paper, but the drawing paper makes a louder sound when lines and curves are drawn.

\subsection{Differences in Sounds Caused by Combinations}

Of all the drawing tools, the loudest sound is generated by marker, followed by pencil with a rounded tip, pencil with a sharp tip, and brush. The overall sound volume gradually decreases in this order. Also, when using a drawing tool other than brush, high quality plain paper has more variations in sound volume while drawing paper is more consistent in terms of loudness. This is considered to be due to the sound of marker hitting the base underneath the surface material such as desk. The sound can get loud because of thinness of the paper.

\section{Experiment 2: Correlation Between Drawing Sounds and Sen- sation of Drawing}

\subsection{Outline of Experiment 2}

An experiment was conducted to verify whether the drawing sounds would increase work efficiency and the real feeling of drawing. The examinees were 13 people, who use computers on a daily basis. In Experiment 2, we examined the changes in the users' sensation of drawing experience by comparing the sounds generated during drawing by six combinations of three kinds of typical drawing tools (brush, pencil, marker) and two kinds of surface materials (quality paper, drawing paper), which were also used in Experiment 1. The participants drawn a certain volume of text in a state where they could not hear any sounds for earplugs and in a state where they could hear their own drawing sounds in a quiet environment. After that, the examinees answered to the questionnaire about the sensation and work efficiency given by the drawing sounds for statistical verification.

\subsection{Combination of Brush and Surface Materials}

\subsubsection{Brush and high-quality plain paper}

Figures 5 and 6 show the questionnaire results carried out after the subject experiment with a combination of brush and high-quality paper. When drawing with a brush on high-quality paper, straight drawn lines are easy to dry quickly. Curvy lines do not dry instantly in areas where lines overlap, and it takes about 5 minutes to dry. A straight line can be drawn about $480 \mathrm{~cm}$ without getting scratchy. It takes a long time for the brush to be worn out completely even after the lines' getting scratchy. However, as the brush tip begins to break up due to the lack of ink, the number of the derivative lines increases, and it becomes difficult to draw. Many experiment participants reported that their work efficiency was not increased very much when no sound was heard. 

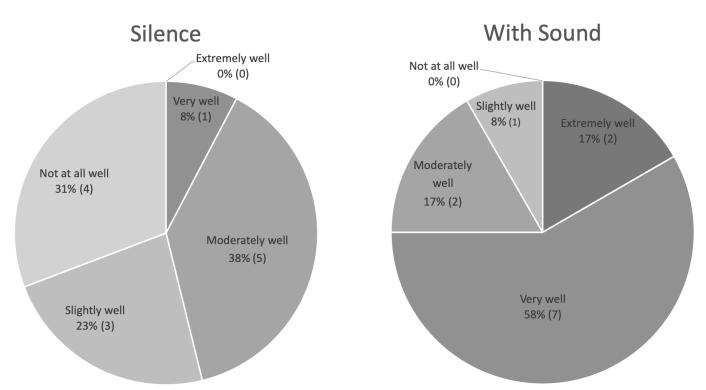

Figure5,6: Questionnaire results regarding work efficiency when using brush and high-quality paper

\subsubsection{Brush and drawing paper}

Figures 7 and 8 show the questionnaire results carried out after the subject experiment with a combination of brush and drawing paper. Drawing with a brush on drawing paper has the characteristic that lines fade and dry quickly. When drawing a straight line, it is possible to draw smoothly about $160 \mathrm{~cm}$. After then, most parts of the line soon fade. Even if a stroke starts with a faint point, it is possible to draw a line reducing the parts that get scratchy by moving the brush slowly. It is difficult to draw the curvy lines with the brush that is rather dry, and the lines become awkward. Since ink is easily absorbed into the drawing paper, we can catch the ink-spreading sound, and the sound of the line's getting scratchy and the sound that the brush itself rubbing against the paper.
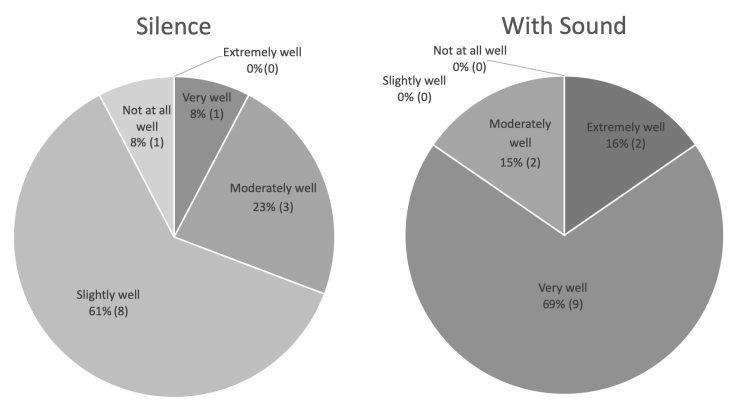

Figure7,8: Questionnaire results regarding work efficiency when using brush and drawing paper

\subsection{Outline of Experiment 2}

\subsubsection{Pencil and high-quality plain paper}

Figures 9 and 10 show the questionnaire results carried out after the subject experiment with a combination of pencil and high-quality paper. When using a sharpened pencil, there is little change in the line weight and the darkness due to writing pressure. When using a pencil with a rounded tip that has been already used to some extent, the darkness changes little. If the writing pressure is strong, the line weight tends to be thick, and when the writing pressure is weak, it tends to be thin. Pencils are hard to be worn out, and people can work for a long time without sharpening them very often. As a result of the experiment, compared with other combinations, there were many participants who grasped the difference in work efficiency in each of the cases: with or without the sound. 

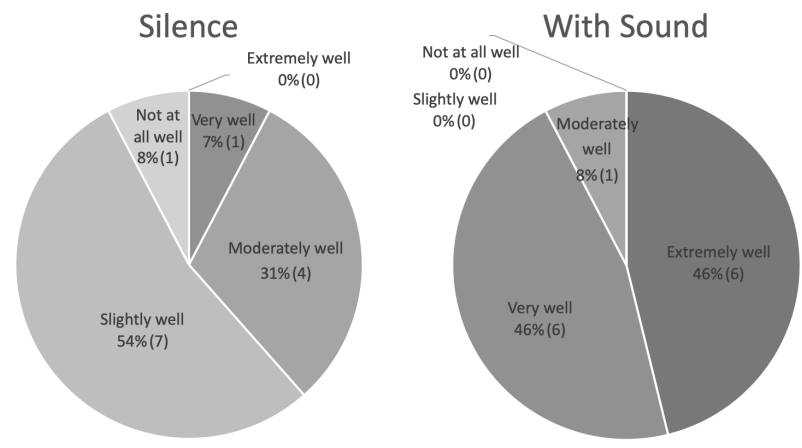

Figure9,10: Questionnaire results regarding work efficiency when using pencil and high-quality paper

\subsubsection{Pencil and drawing paper}

Figures 11 and 12 show the questionnaire results carried out after the subject experiment with a combination of pencil and drawing paper. When drawing with a pencil on drawing paper, the "real feel" of drawing is most easily obtained. Because of the thickness of the paper, it is easy to draw, and the line darkness can be clearly changed by the writing pressure. When straight lines are repeatedly drawn on an A3 size of drawing paper with the just sharpened pencil, the tip will have to be rounded after about $3000 \mathrm{~cm}$ drawing that deserves about 1 and a half paper. As the pencil is rounded, the drawn lines gradually get thicker. As a result of the experiment, many participants reported that their work efficiency was increased when there was the sound of this combination compared to other combinations.
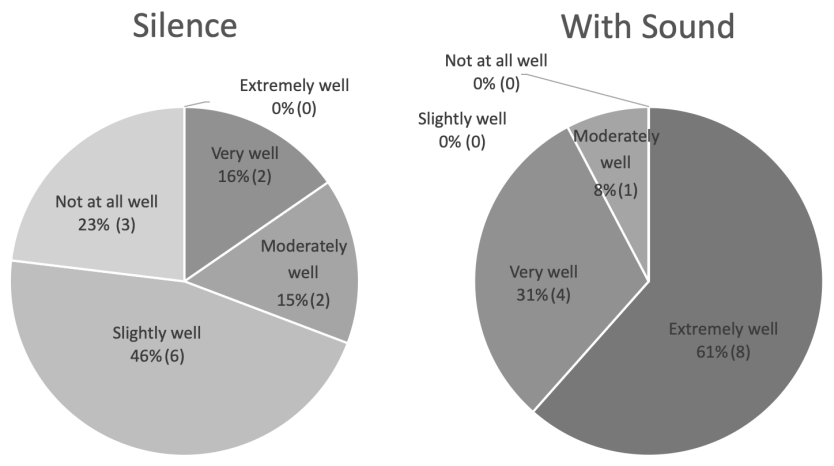

Figure1 1,12: Questionnaire results regarding work efficiency when using pencil and drawing paper

\subsection{Combination with Marker and Surface Materials}

\subsubsection{Marker and high-quality plain paper}

Figures 13 and 14 show the questionnaire results carried out after the subject experiment with a combination of marker and high-quality paper. When drawing with a marker on highquality plain paper, the ink spreading way differs depending on each of the cases to draw a point or drawing a line. The color appears more clearly and vividly when drawing a point than drawing a line. Although it depends on the shape of the tip, the surface where the maker and the paper come into contact is reduced due to subtle changes in the user's individual holding and writing way. This can lead to inability of smooth drawing. As a result of the experiment, majority of the people answered that their work efficiency was improved with the sound, but there were also many ambiguous answers: "Neither." 

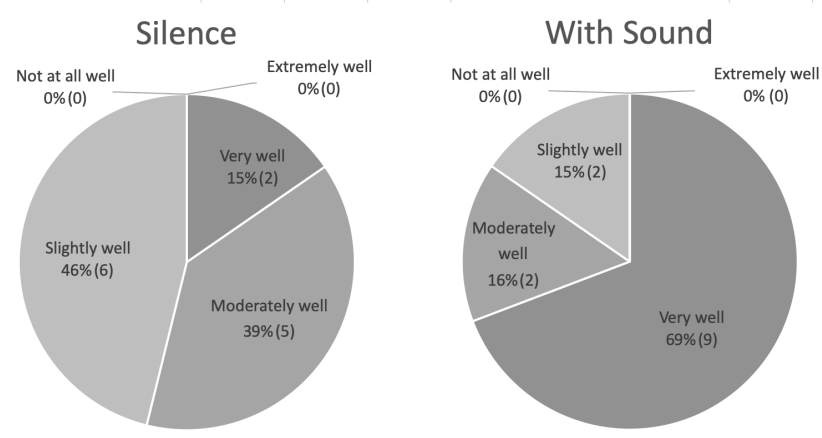

Figure13, 14: Questionnaire results regarding work efficiency when using marker and high-quality paper

\subsubsection{Marker and drawing paper}

Figures 15 and 16 show the questionnaire results carried out after the subject experiment with a combination of marker and drawing paper. When drawing with a marker on drawing paper cause a strong sensation that ink is being absorbed into drawing paper. In many cases, "ink-spread" may occur depending on the drawing to overlap the lines like curvy lines or the pausing moment for which we are just holding the marker in contact with the paper. There are various marker shapes, for example, types of a rounded tip and a rectangular tip. No matter what kind of marker is used, differences in points and lines are likely to occur due to subtle changes of holding and drawing way, and irregular lines tend to be born. As a result of the experiment, as with the one for marker and high-quality paper, opinions were relatively distributed. In addition, there were some opinions that the work efficiency was not improved because the participants originally disliked the sound generated by marker and the drawing paper.
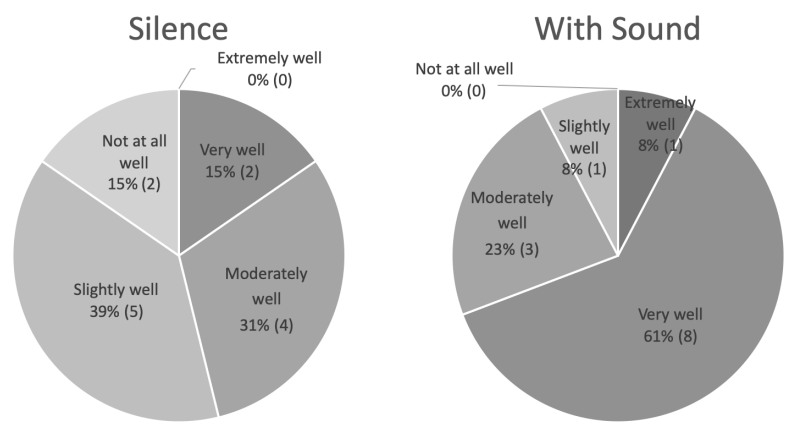

Figure15, 16: Questionnaire results regarding work efficiency when using marker and drawing paper

\section{Conclusion and Future Work}

\subsection{Conclusion}

In recent drawing software, there are many types of products, and it is possible to use them properly depending on the different purposes. However, the variety is only limited to the touches 
of the finished works on the screen and the output of the works. Naturally, we hear various sounds in our lives. Since usually people are aware of sounds almost unconsciously, it may be difficult to get public empathy for the phenomena that the sound changes depending on the combination of drawing tools and surface materials during drawing. However, by the experiments in this paper, it was clear that there was a change of the waveforms depending on the combination of the drawing tools and the surface materials. In addition, we were able to capture the limitations how long the tools can be utilized by the combinations and each combination's characteristic. Based on this, we propose that the factor "sounds", which is taken for granted in our everyday lives, provide sensation of drawing for users without the feel of complexity. Incorporating "analog-like reality" into the system can be achieved in this way, instead of suggesting new works and specifications in drawing software.

\subsection{Future Work}

The experiments conducted in this paper were just some examples, and other combinations of drawing tools and surface materials are possible. There are also prospective combinations using drawing tools such as chalk and crayon, and surface materials such as Japanese paper and blackboard, which are previously mentioned in the first half of this paper. Out of them, some combinations would not enable users to draw properly. In the future, we will explore and examine the factors that give us a sense of reality from a different point of view besides "sounds" featured here. We will also proceed with the further implementation of "sounds that provide the realistic experience of drawing" through the development and the subject experiments based on this paper.

\section{References}

[1] Yoshizumi Yokokura, "Stereo production technology, stereo CM production, the actual creation of commercial sound effects [in Japanese]", The journal of the Institute of Television Engineers of Japan, 34(4), 317-319, 1980.

[2] Kenichi Fujimoto, "Charm of analog record (4) Charm of single board[Japanese]", Impact Publishing, Ambos Mundos (4), 96-98, 2000- 01.

[3] Noriko Ohisa, Katsumi Yoshida, Tomohisa Yanbe, Mitsuo Kaku, "Effect of autonomic nervous system activity while listening to music", The Autonomic nervous system 42(3), 265-269, 2005.

[4] Sachiko Deguchi, Naoya Furuta, "A Study on the Generation of Sounds and Figures using LCD Tablet”, IPSJ SIG Technical Report, 115(2), 2005

[5] Eishi Sugasawa, "Image editing software for creative use (5) Make picture letters with paint tools [Japanese]”, Nikkei PC (501), 133-136, 2006-03-13.

[6] Kenichi Furukawa, "Development of Pocket Painting Tool using USB memory", Research report of JET Conferences, 2006(6), 65-68, Dec 2006.

[7] Shinya Kasai, Yukinori Goto, Yuko Murayama, "e-gakki : This system convert Drawing Information to Sound", The 69th National Convention of IPSJ, 2007(1), 487-488, Mar 2007. 
[8] Asako Kimura, Hideyuki Omachi, Fumisa Shibata, Hideyuki Tamura, "A Study for Presentation of Tactile Sensation with Sound Feedback to Touch Sensor", Information Processing Society of Japan (IPSJ) Human-Computer Interaction (HCI), 2007(68(2007-HCI-124)) 9-16, 2007.

[9] Yuta Katsutani, Yukari Nagai, Junya Morita, "Influence of Music on Drawing", Bulletin of Japanese Society for Science of Design, 59(0), 59, 2012.

[10] Takayuki Fujimoto, "Toward Information Design 3.0: The Information Design for 'Communicate", Building maintenance \& management, vol. 34, pp. 42-46, 2013.

[11] Takayuki Fujimoto, “Understandability Design : what is 'Information Design'?”, The journal of Information Science and Technology Association, vol. 65, pp. 450-456, 2015.

[12] Satomi Kato, Shinji Mizuno, "Improvement of the Analyzing Method for Drawing and Sound Generation System "RAKUGACKY"'”, Digital Contents Creation, 5, 11-19, 2017.

[13] Ziran Fan, Takayuki Fujimoto, "Implementation and Evaluation of Document Production Support System "DLO-Editor" with Obsession Mechanism", 25th International Conference on Systems Engineering, Aug 2017.

[14] Ziran Fan, Takayuki Fujimoto, "Implementation of Document Production Support System with Obsession Mechanism", 4th International Conference on Computational Science/ Intelligence \& Applied Infomatics(CSII2017), July 2017.

[15] Takayuki Fujimoto, "Ideology of AoD: Analog On Digital-Operating Digitized Objects and Experiences with Analog-like Approach", 1st International Conference on Interaction Design and Digital Creation / Computing (IDDC 2018), July 2018.

[16] Yui Tanaka, Takayuki Fujimoto, "A Design of Application to Turn a Smartphone into a Computer Mouse and Possibility of Preventing from Being Copied", 1st International Conference on Interaction Design and Digital Creation / Computing (IDDC 2018), July 2018.

[17] Ziran Fan, Takayuki Fujimoto, "Proposal of a Design Method to Apply the Analog Features to Digital Media", The 2nd International Conference on Applied Cognitive Computing, Aug 2018.

[18] Ziran Fan, Takayuki Fujimoto, "Proposal of a Scheduling App Utilizing Time-Perception-Reality in Analog Clocks", 1st International Conference on Interaction Design and Digital Creation / Computing (IDDC 2018), July 2018.

[19] Ziran Fan, Takayuki Fujimoto, "Proposal of a Digital Book Application that Offers Analog-like Usability", The 15th IEEE Transdisciplinary- Oriented Workshop for Emerging Researchers, pp. 26, 2018.

[20] Ziran Fan, Takayuki Fujimoto, "Method to Control Children's Smartphone Use Based on the Motif of Analog Fuel System", ICSTR Bangkok - International Conference on Science \& Technology Research, 2018. 